\section{Molecular and Multimodality Imaging in Cardiovascular Disease}

T.H. Schindler, R.T. George, and J.A.C. Lima, Eds.

New York, NY: Springer, 2015, 247 pages, $\$ 159$

Dr. Schindler and 28 expert coauthors have written a 12-chapter textbook on molecular and multimodality imaging of cardiovascular disease. The text provides a well-written discussion of the technical characteristics and clinical applications of advanced cardiac imaging modalities, including PET, MR imaging, CT, hybrid PET/MR imaging, and hybrid PET/CT.

The first two chapters are devoted to technical aspects of PET/ MR imaging, with a clear discussion of use of the Dixon sequence to derive attenuation maps for the PET data. The authors present examples of myocardial ischemia, scarring, inflammation, and cardiac involvement in both metastatic angiosarcoma and vascular inflammation to demonstrate the synergy between ${ }^{18}$ F-FDG PET and late-gadolinium-enhancement MR imaging as markers of disease. The third chapter adds an analysis of MR myocardial perfusion measurements, as well as the value of MR imaging with late gadolinium enhancement, to distinguish between acute inflammation in the region of an acute myocardial infarction and viable ischemic myocardium. Chapter 4 addresses the evaluation of cardiac sarcoidosis by such methods as echocardiography, ${ }^{67} \mathrm{Ga}$ imaging, and-in an in-depth discussion- ${ }^{18}$ F-FDG PET/CT. Chapter 5 covers cardiac amyloidosis, with a balanced presentation of the value of echocardiography and of radionuclide imaging with ${ }^{99 \mathrm{~m}} \mathrm{Tc}$-phosphonates and ${ }^{18} \mathrm{~F}$-florbetapir. Chapter 6 describes the approaches to detecting viable ischemic myocardium with echocardiography, ${ }^{18}$ F-FDG PET/CT, perfusion PET/CT, ${ }^{201} \mathrm{Tl}$ redistribution SPECT, and MR imaging. Chapter 7 tackles the role of MR imaging in cardiovascular risk assessment based on changes in T1 signal, extracellular volume fraction, and late gadolinium enhancement. Chapter 8 , which focuses on multimodality imaging of atherosclerotic plaque, provides an excellent, concise review of the evolution of atheroma, as well as a description of the potential clinical role of PET/CT with ${ }^{18} \mathrm{~F}-\mathrm{FDG}$ or ${ }^{18} \mathrm{~F}$-fluoride to localize and characterize these lesions. Chapter 9 describes the use of CT coronary angiography to evaluate coronary lesion morphology and myocardial perfusion. Chapter 10 concentrates on the clinical value of a fused 3-dimensional display of CT coronary angiography and myocardial perfusion to define the relationship of anatomy to physiology. Chapter 11 discusses the potential long-term clinical value of combining CT coronary angiography with measures of myocardial flow reserve, especially in the evaluation of noncalcified, nonobstructive plaque. Chapter 12 summarizes the added clinical value of combining data on coronary calcification measurement with myocardial perfusion imaging for risk stratification. In patients with normal myocardial perfusion results, the information from coronary calcium scoring adds significant longterm prognostic data.

Overall, this well-illustrated and well-referenced text constitutes a concise update on multimodality cardiac imaging that will be of value to practitioners, trainees, and investigators.

\section{H. William Strauss \\ Memorial Sloan Kettering Cancer Center 1275 York Ave., Room S-212 \\ New York, NY 10021 \\ E-mail: straussh@mskcc.org}

Published online May 12, 2016 DOI: 10.2967/jnumed.116.178129 